\title{
Utilisation de la signature isotopique des radionucléides relâchés par les mines et les usines de traitement de minerais d'uranium, pour discriminer aux bas niveaux leur impact environnemental de celui de la radioactivité tellurique naturelle
}

\author{
P. ZETTWOOG*, N. LEMAÎTRE*, S. BERNARD**, Y. VAUZELLE**
}

(Manuscrit rę̧u le 6 janvier 1997, révisé le 22 avril 1997, accepté le 30 août 1997)

RÉSUMÉ Dans la perspective du contrôle réglementaire de l'impact environnemental des sites d'extraction et de traitement des minerais d'uranium, l'Office de Protection contre les Rayonnements Ionisants (OPRI) et Algade développent des méthodes de différentiation entre les phénomènes d'origine naturelle et ceux ayant pour origine une pratique industrielle. Il a été établi que sur ces sites la signature isotopique de l'uranium, du radium et du radon d'origine minière se distingue nettement de celle des mêmes éléments présents naturellement dans l'écosphère locale. Il en résulte une possibilité de discrimination entre les doses efficaces d'origine minière et celles qui sont à associer à la radioactivité tellurique non perturbée. Des procédures d'exploitation des résultats des réseaux de surveillance réglementaires des installations minières, fondées sur ces méthodes, sont envisageables. Elles pourraient permettre la vérification du respect de la réglementation dans les situations où les doses efficaces d'origine minière sont inférieures aux doses efficaces dues à la radioactivité tellurique et à leurs fluctuations.

ABSTRACT Use of isotopic signature of radionuclides released from uranium mines and mills to discriminate low levels of environmental impact against natural background levels.

In view of the regulatory assessment of the radiological impact of uranium ores mining and milling site, the Office de Protection contre les Rayonnements Ionisants (OPRI) and Algade are developing methods which allow the differentiation between natural phenomena and man-made phenomena. It has been recognized that for a region where mining activities took place, the isotopic

* Office de Protection contre les Rayonnements Ionisants, 78110 Le Vésinet, 31 rue de l'écluse, BP 35, France.

** ALGADE, 87250 Bessines-sur-Gartempe, BP 46, France. 
signature of uranium, radium and radon can clearly be recognized from the same product of natural origin. For exemple in the case of radon the industry produces only ${ }^{222} \mathrm{Rn}$ while the natural emanations are composed of ${ }^{220} \mathrm{Rn}$ and ${ }^{222} \mathrm{Rn}$. In the case of radium the industry produces only ${ }^{226} \mathrm{Ra}$ while both ${ }^{226} \mathrm{Ra}$ and ${ }^{228} \mathrm{Ra}$ are present in the natural environment. Demonstration of completion with regulatory limits in situations where the industrial impact is less than the impact from the natural radioactive environment could be made feasible on a routine basis.

\section{La réglementation française relative à la protection de l'environnement des sites d'extraction de minerais d'uranium}

Le besoin d'une méthode de discrimination entre les impacts miniers et l'impact de la radioactivité tellurique naturelle est apparu à la suite de l'augmentation de la sévérité de la réglementation européenne sur la protection des population contre les rayonnements ionisants.

\subsection{Historique}

En France, le document réglementaire qui concerne spécifiquement la protection de l'environnement des mines d'uranium, des usines de traitement des minerais, et des installations de stockage des déchets miniers et des résidus solides du traitement est le décret $n^{\circ}$ 90-222 du 9 mars 1990. Ce décret a été publié au Journal Officiel de la République française du 13 mars 1990. Ce document est introduit dans le règlement général des industries extractives institué par le décret $n^{\circ} 80-331$ du 7 mai 1980.

Ce document entérine pour l'essentiel les dispositifs de contrôle des rejets et de mesure de l'exposition de la population aux rayonnements ionisants que les producteurs avaient mis progressivement en place de leur propre initiative dès le début des années 70 , se référant pour la conception de ces dispositifs aux recommandations des organisations internationales de l'époque.

Les rédacteurs du décret $n^{\circ}$ 90-222 ont pris entre autres pour référence le document AIEA SS n 43 de 1975 qui fixait les objectifs des réseaux de surveillance, ainsi que la publication CIPR $n^{\circ} 43$ (1984) de 1984. Cette dernière publication confirmait la justesse des objectifs du document $S S n^{\circ} 43$, précisait quelques concepts importants, dont celui de "groupes critiques de la population », déjà introduit dans la publication CIPR $n^{\circ} 26$ (1977). Pour mesurer les doses efficaces de ces groupes critiques deux types de stratégies de surveillance étaient proposées,

- celles fondées sur la mesure des quantités de radionucléides rejetés (mesure des termes "source"), suivie de la modélisation des transferts environnementaux et des doses efficaces, 
- celles qui visent directement à la mesure ou la détermination des quantités des différents radionucléides inhalées et ingérés par les groupes critiques de la population, dont on déduit les doses efficaces.

\subsection{Les points clés du décret $n^{\circ} 90-222$}

Le décret $\mathbf{n}^{\circ}$ 90-222 impose la limitation de la fraction de la dose efficace annuelle qui est à attribuer aux opérateurs miniers. Il retient, pour la démonstration du respect de cette limite, la deuxième des stratégies ci-dessus, car c'est la seule qui soit applicable dans le cas des sites miniers, compte tenu de ce que l'opérateur minier ne peut pas mesurer en routine les termes « source » relatifs aux installations de stockage des déchets miniers et des résidus radifères.

Le décret introduit le concept de « groupes critiques de la population ». Les « groupes critiques de la population » sont caractérisés lors de l'étude radiologique du site, compte tenu des sources et des voies de transfert critiques relatives aux radionucléides significatifs pour la radioprotection. Le réseau de surveillance est alors mis en place. Des arrêtés préfectoraux spécifiques précisent la fréquence et l'emplacement des points de prélèvement dans l'environnement et les méthodes de mesures à utiliser.

En ce qui concerne la radioactivité contenue dans les eaux d'exhaure des mines et les effluents liquides des usines, le décret introduit le principe d'optimisation de la radioprotection. Les limites de rejet sont plus ou moins sévères en fonction de la dilution apportée par le cours d'eau récepteur et des voies de transfert potentielles. Si une station d'épuration du radium ou de l'uranium est exigée, l'opérateur est tenu de mettre en œuvre la meilleure technologie existante conduisant à un rejet aussi faible que possible.

\subsection{Limites de dose efficace annuelle introduites dans la circulaire d'application}

La circulaire d'application du décret $n^{\circ} 90-222$ a fixé la limite à respecter en ce qui concerne la dose efficace ajouté par l'industriel à la dose efficace d'origine naturelle pour les mêmes radionucléides et les mêmes voies d'exposition. Sauf cas particulier la dose efficace est déterminée en prenant en compte l'exposition aux rayonnements gamma, l'inhalation des descendants à vie courte des isotopes du radon et des émetteurs alpha à vie longue des poussières radioactives, l'ingestion d'uranium et de radium avec la consommation de l'eau du site et des produits alimentaires locaux.

Cette limite annuelle de dose efficace pour les personnes des groupes critiques est égale à $5 \mathrm{mSv}$. Dans le calcul on admet que $5 \mathrm{mSv}$ correspond, entre autres voies d'exposition, à l'inhalation d'une énergie alpha potentielle (EAP) de $2 \mathrm{MJ}$ en ce qui concerne les descendants du ${ }^{222} \mathrm{Rn}$, ou à l'ingestion de $7000 \mathrm{~Bq}$ de ${ }^{226} \mathrm{Ra}$. 
Dans un avenir proche le texte de la circulaire d'application sera mis en conformité avec la limite de dose efficace de $1 \mathrm{mSv}$ par an en moyenne sur 5 ans retenue par la Directive 96/29 Euratom des Communautés européennes fixant les normes de base relatives à la protection sanitaire. À la dose efficace de $1 \mathrm{mSv}$ correspond l'inhalation de 0,73 MJ d'EAP des descendants du ${ }^{222} \mathrm{Rn}$. Cette quantité est inhalée par une personne exposée un an à une concentration de ${ }^{222} \mathrm{Rn}$ à l'équilibre de $18 \mathrm{~Bq} \mathrm{~m}^{-3}$, la concentration en Energie Alpha Potentielle étant de environ $100 \mathrm{~nJ} \mathrm{~m}^{-3}$. A $1 \mathrm{mSv}$ correspond également l'ingestion de $3 \times 10^{6} \mathrm{~Bq}$ de ${ }^{226} \mathrm{Ra}$, ou de $8,3 \times 10^{5} \mathrm{~Bq}$ de ${ }^{210} \mathrm{Po}$.

On notera que la Directive 96/29/Euratom des Communautés européennes fixant les normes de base relatives à la protection sanitaire utilise le terme «groupe de référence» en remplacement du terme "groupe critique ».

\section{Problèmes rencontrés par les opérateurs dans la mise en application du décret $\mathrm{n}^{\circ} \mathbf{9 0 - 2 2 2}$}

\subsection{Situation actuelle (1997) : limite annuelle de dose efficace de $5 \mathrm{mSv}$}

Les opérateurs ont accepté après discussion et mis en œuvre les dispositions retenues dans les arrêtés préfectoraux en ce qui concerne le contrôle des rejets et les réseaux de surveillance. Il s'avère que la valeur de l'équivalent de dose efficace correspondant au cumul des expositions « industrielles » et des expositions «naturelles » est en général inférieure à la limite annuelle de $5 \mathrm{mSv}$.

Pour restituer la contribution d'origine «industrielle » 1'opérateur soustrait de la valeur cumulée mesurée une valeur forfaitaire correspondant au « bruit de fond naturel $\gg$.

Le respect de la limite étant acquis même avec les valeurs cumulées, l'opérateur accepte d'être conservateur en ce qui concerne la détermination de ce bruit de fond naturel. Pour être sûr de ne mesurer que du «naturel », il mesure relativement loin des sources industrielles. Mais les résultats de telles mesures sont plus représentatifs du bruit de fond régional (c'est à dire au delà de quelques kilomètres des sources de radioactivité du site minier surveillé), que du bruit de fond local à quelques centaines de mètres de ces sources. Il est fréquent que le bruit de fond local soit plus élevé que le bruit de fond régional. La méthode de soustraction, dans les conditions où elle est actuellement pratiquée, a conduit à des surévaluations sciemment acceptées de la composante industrielle.

\section{Situation attendue : limite annuelle de dose efficace de $1 \mathrm{mSv}$}

Sur les sites miniers en exploitation, on peut aboutir, rien que par l'inhalation de l'EAP des descendants du radon 222 émis par dépôts de résidus de 
traitement des minerais, à une dose efficace supérieure à $1 \mathrm{mSv}$ par an pour certains des groupes de la population. Respecter pour ces groupes critiques la limite annuelle d'équivalent de dose efficace d'origine industrielle de $1 \mathrm{mSv}$, (avec la correspondance indiquée ci-dessus pour le ${ }^{222} \mathrm{Rn}$ ), ne sera éventuellement pas possible dans le cas de certains dépôts de résidus de traitement exploités et non encore recouverts.

Sur les sites miniers réhabilités, les sources de ${ }^{222} \mathrm{Rn}$ sont maîtrisées et la dose efficace due à l'inhalation de l'EAP est ramenée à moins de $1 \mathrm{mSv}$ par an. Mais l'EAP n'est pas seule en cause. La dose efficace « industrielle » obtenue en cumulant toutes les voies d'exposition et tous les radionucléides peut dépasser la limite de dose efficace de $1 \mathrm{mSv}$ pour certains groupes de référence.

La dose efficace d'origine «naturelle » de son côté s'établit dans la gamme allant de 1 à $3 \mathrm{mSv}$ pour l'ensemble de la population.

Le réseau de surveillance ne peut fournir que la somme des doses d'origine « industrielle » et "naturelle ». Si après soustraction des «bruits de fond naturel » on établit qu'il y a des dépassements de la limite, l'opérateur est tenu de mieux épurer ses rejets et d'améliorer ses confinements. Avant de mettre en œuvre les techniques adéquates, il demandera toutefois à être convaincu que la contribution "industrielle »n'a pas été surévaluée par une estimation trop basse de la contribution «naturelle».

L'opérateur constate que la contribution «naturelle » est de l'ordre de la limite réglementaire, et est souvent supérieure à la contribution industrielle réglementée. Il va chercher à établir sans conservatisme les valeurs du bruit de fond naturel avant de les retrancher des valeurs cumulées. Pour ce faire il va s'attacher à rétablir avec la précision suffisante les valeurs du bruit de fond local avant sa perturbation par ses rejets et relâchements. Il devra solidement argumenter les valeurs proposées.

L'introduction d'une limite plus sévère a ainsi fait apparaître un problème que personne jusqu'ici ne cherchait à résoudre, celui de la discrimination des contributions des sources « naturelles » et des sources « industrielles » dans le cumul des doses efficaces.

Le besoin de cette discrimination est apparue en premier pour l'inhalation de l'EAP des descendants du radon 222. Dans un site vallonné la charge en EAP de l'atmosphère, qui résulte des exhalaisons de radon 222 des sols locaux non contaminés, est très variable à l'échelle de quelques centaines de mètres, c'est à dire d'une station du réseau à l'autre.

Cette variabilité s'explique par la variabilité des caractéristiques pédologiques des sols et de leur teneur en radium, par l'orographie locale et par les situations micrométéorologiques locales. Des valeurs élevées obtenues sur une 
station n'indiquent pas nécessairement le marquage par le radon 222 d'origine industriel. Il peut s'agir d'une station installée dans un emplacement mal ventilé. C'est pourquoi depuis longtemps nous considérons que la référence à un bruit de fond régional uniforme n'est pas adéquate pour l'établissement de l'EAP « industrielle » dans les sites vallonnés.

De même, la charge radioactive d'origine naturelle des eaux souterraines et des eaux de surface observable à proximité immédiate du site minier correspond à celle d'une formation géologique qui a hébergé une forte anomalie d'uranium. Cette charge radioactive d'origine naturelle peut être très différente de celle qui est observée au niveau des aquifères d'un bassin versant voisin mais n'ayant jamais hébergé de gisement d'uranium.

Il est donc nécessaire de trouver une méthode de discrimination des deux contributions qui soit basée sur autre chose que des mesures du bruit de fond naturel non représentatives de la zone marquée.

\section{Discrimination fondée sur la différence des signatures isotopiques}

L'Office de protection contre les rayonnements ionisants, (OPRI) organisme technique du Ministère de la Santé, qui a notamment pour mission la mesure de l'exposition des populations tant d'origine naturelle qu'industrielle ou médicale, se préoccupe de développer, en collaboration avec la société Algade, une filiale de services de la Cogema spécialisée dans la surveillance des sites miniers, une méthode permettant de fonder la discrimination de chacune de ces deux composantes sur une base méthodologique scientifiquement acceptable. La possibilité de discriminer les charges environnementales en radionucléides d'origine naturelle et celles d'origine industrielle à partir de leur signature isotopique est explorée.

\subsection{Principe de la méthode de la signature isotopique}

Le principe de la méthode est simple.

Les concentrations atomiques des isotopes radioactifs des chaînes de ${ }^{238} \mathrm{U}$, ${ }^{235} \mathrm{U}$ et ${ }^{232} \mathrm{Th}$, (éléments uranium, thorium, radium, radon, polonium, bismuth et plomb), mesurées par le réseau de mesure dans les différents compartiments physiques, (atmosphère, eaux de surface et eaux souterraines, sols et sédiments, chaîne alimentaire), ne sont en général pas du tout uniformes d'un point à l'autre du domaine. Par définition, la signature isotopique de la radioactivité naturelle est l'ensemble des rapports de ces concentrations. La non uniformité des concentrations isotopiques n'exclut pas l'uniformité de leurs rapports. La méthode de la signature isotopique est susceptible de fonctionner lorsque, à l'échelle du 
domaine surveillé, au moins pour certains milieux, la signature isotopique de au moins certains des radionucléides d'origine naturelle est uniforme.

Le domaine surveillé recouvre le domaine où l'impact industriel existe vraisemblablement, mais aussi des zones périphériques suffisamment éloignées pour qu'il soit certain qu'elles soient représentatives du bruit de fond naturel. On établit la signature isotopique des radionucléides d'origine naturelle dans ces zones périphériques pour tous les milieux pris en compte dans le calcul des doses efficaces. On fait l'hypothèse que cette signature est également celle des radionucléides d'origine naturelle du domaine où il y a marquage par les sources industrielles.

Dans le cas des rejets des installations minières françaises, il se trouve que le rapport de l'activité des isotopes de respectivement la chaîne de ${ }^{238} \mathrm{U}$ et celle de ${ }^{232}$ Th est supérieur à 100 . Il ressort de la figure 1 que le rapport de l'activité de ${ }^{238} \mathrm{U}$ à celle de ${ }^{232} \mathrm{Th}$ dans les roches de la croûte terrestre est dans la gamme 0,4 à 10 .

La signature isotopique des marquages dus aux sources industrielles est donc caractérisée par des valeurs quasi-nulles des concentrations des isotopes $\mathrm{du}{ }^{232} \mathrm{Th}$ vis-à-vis de celles des isotopes de la chaîne de ${ }^{238} \mathrm{U}$. Il en résulte que, à partir des concentrations des isotopes de la chaîne du thorium mesurées dans chaque station de la zone d'impact et de la signature isotopique mesurée dans les stations des zones sans impact, on sait reconstruire les concentrations des isotopes de la chaîne de ${ }^{238} \mathrm{U}$ d'origine naturelle de chaque station de la zone d'impact.

L'hypothèse de l'uniformité se vérifie lorsque les concentrations en uranium et en thorium des sols naturels et des formations géologiques d'une part, les processus de lessivage et de transport des radionucléides dans les milieux récepteurs d'autre part, varient peu à l'échelle du domaine surveillé par les réseaux de mesure.

\section{2. État d'avancement des travaux de qualification de la méthode}

Notre intention est d'étudier dans quelle mesure la méthode de la signature isotopique peut résoudre le problème posé, et les conditions de la validité des hypothèses associées dans le cas des sites miniers français. Dans les cas où la situation orographique est simple, comme dans les plaines argilosableuses et argilo-calcaires du Bassin parisien, la méthode fonctionne. Cependant les concentrations des radionucléides d'origine naturelle sont à peu près uniformes en valeurs absolues sur tout le domaine, et la simple soustraction du bruit de fond uniforme restitue tout aussi bien la composante industrielle. En fait, dans la plupart des sites miniers français, la situation de la radioactivité naturelle est moins triviale, le bruit de fond n'est pas uniforme d'un bout à l'autre du domaine en ce qui concerne les concentrations 
des radionucléides d'origine naturelle. Cependant le rapport des concentrations, c'est à dire la signature isotopique, peut rester uniforme. Identifier les sites pour lesquels la méthode de restitution à partir des signatures isotopiques est valable est notre objectif.

Nous présentons les recherches en cours et les résultats obtenus. En ce qui concerne l'impact des rejets atmosphériques du ${ }^{222} \mathrm{Rn}$, la méthode nous parait validée pour au moins trois sites caractérisés par des reliefs significatifs sur le plan de la dispersion (différence d'altitude de quelques centaines de mètres, existence de plusieurs cours d'eau, rugosité du sol variable). En ce qui concerne l'impact des rejets et des relâchements de radium et d'uranium dans les aquifères, nous en sommes au stade des investigations préliminaires.

\section{Résultats obtenus pour la restitution de l'impact ${ }^{222} \mathbf{R n}$ industriel}

Les réseaux de mesure de 1'impact radiologique du ${ }^{222} \mathrm{Rn}$ industriel exploités par Algade mesurent la valeur moyenne sur le mois des concentrations atomiques du ${ }^{222} \mathrm{Rn}$ et de ses descendants à vie courte porteurs de l'EAP, $\left({ }^{218} \mathrm{Po}\right.$, ${ }^{214} \mathrm{~Pb}$ et ${ }^{214} \mathrm{Bi}$ ), ainsi que celle du ${ }^{212} \mathrm{~Pb}$, dont on déduit celle du ${ }^{212} \mathrm{Bi}$, qui descendent l'un et l'autre de ${ }^{220} \mathrm{Rn}$. Il est ainsi possible de calculer chaque mois pour chaque station du réseau des zones périphériques le rapport $A$ de la concentration en atomes de ${ }^{222} \mathrm{Rn}$ à la somme des concentrations en atomes de ${ }^{212} \mathrm{~Pb}$ et de ${ }^{212} \mathrm{Bi}$ d'origine naturelle. Les atomes de ${ }^{212} \mathrm{~Pb}$ et de ${ }^{212} \mathrm{Bi}$ ont remplacé nombre pour nombre les atomes de ${ }^{220} \mathrm{Rn}$ exhalés quelques minutes plus tôt par les sols.

Le rapport $A$ d'une station est donc interprété dans la suite comme se rapportant au rapport des exhalaisons des atomes de ${ }^{222} \mathrm{Rn}$ et de ${ }^{220} \mathrm{Rn}$, ces exhalaisons ayant été pondérées des décroissances radioactives respectives et des effets de la dispersion atmosphérique pendant le transfert entre les sols et cette station. Dans les régions uranifères françaises le poids des exhalaisons du domaine surveillé, avec des temps de transfert inférieurs à l'heure, excède en général celui des exhalaisons aux échelles régionales et continentales, dont la contribution devient négligeable dès que les temps de transferts sont supérieurs à la journée.

Dans la référence (Bernhard et al., 1995), on examine les conditions pour lesquelles on peut, en théorie, espérer obtenir la constance du rapport $A$ des concentrations en atomes de ${ }^{222} \mathrm{Rn}$ et des concentrations en atomes de ${ }^{212} \mathrm{~Pb}$ et ${ }^{212} \mathrm{Bi}$ dans l'air échantillonné par l'ensemble des stations du réseau de surveillance. 
Dans la pratique nous avons fondé la validité de l'application de la méthode sur une faible dispersion des valeurs mesurées de $A$ pour un nombre suffisant de stations de fond couvrant bien le domaine surveillé, contenant en particulier des stations mal ventilées avec des charges radon fortes, et des stations bien ventilées avec des charges radon faibles.

Si l'écart-type relatif $\triangle A / A$ des valeurs de $A$ est faible (le critère d'applicabilité est $\triangle A / A<30 \%$ ), on calcule un rapport $\langle A>$ qui est la moyenne des valeurs de $A$ obtenues sur les stations non marquées du domaine. Les concentrations en atomes de ${ }^{212} \mathrm{~Pb}$ et ${ }^{212} \mathrm{Bi}$ étant mesurées également dans les stations où il y a un marquage par du radon 222 industriel, on déduit les concentrations en radon 222 d'origine naturelle en y appliquant le facteur $\langle A\rangle$.

Le tableau I montre les valeurs du rapport $\langle A>$ que nous avons obtenues en moyenne annuelle sur un certain nombre de sites en France et en quelques pays d'Afrique. Dans le cas des contextes sédimentaires, les valeurs de $<A>$ de ce tableau sont dans la gamme 300-380, le cas du Languedoc, avec des épisodes volcaniques, sortent de cette gamme avec $\angle A>=860$. Dans le cas des contextes cristallins, les valeurs de $\langle A\rangle$ s'étalent de 500 à 5200 .

Dans un schéma primaire qui ne tient pas compte ni des processus de diffusion des atomes de radon dans l'espace des pores du sol précédant leur exhalaison, ni des dépôts au sol des aérosols atmosphériques porteurs de descendants du radon, le rapport du flux atomique de radon 222 au flux atomique de radon 220 est égal à :

$$
\frac{\left[\mathrm{Ra}_{226}\right] E_{222} \sqrt{T_{222}}}{\left[\mathrm{Ra}_{224}\right] E_{220} \sqrt{T_{220}}}
$$

avec $\left[\mathrm{Ra}_{226}\right],\left[\mathrm{Ra}_{224}\right]$, activités massiques des isotopes du radium dans le sol, $E_{222}, E_{220}$, coefficients d'émanation des isotopes du radon hors des matrices cristallines,

$T_{222}, T_{220}$, périodes radioactives des isotopes 222 et $220 \mathrm{du}$ radon.

La valeur du rapport des racines carrées des périodes est de 77 . Le rapport des coefficients d'émanation est de l'ordre de 1 sauf particularités minéralogiques. Dans la croûte terrestre, les activités des isotopes du radium sont en général en équilibre radioactif avec celles de leurs tête de chaîne. Des écarts à l'équilibre sont cependant rencontrés dans les sols.

Les valeurs attendues pour $A$ en se basant sur ce schéma, et sur les données de la figure 1 pour les valeurs ${ }^{238} \mathrm{U} /{ }^{232} \mathrm{Th}$, et en admettant l'équilibre des isotopes du radium et de leur tête de chaîne, sont donc dans la gamme allant de $77 \times 0,4=30$, à $77 \times 10=770$. Les valeurs de $\langle A>$ du tableau I, dans la gamme 


\section{TABLEAU I}

Rapports $\langle A\rangle$ obtenus sur quelques réseaux équipés du dosimètre alpha spectral de ALGADE (Valeurs établies en moyenne annuelle).

Ratios $\langle A\rangle$ obtained over some networks equipped with the ALGADE alpha spectral dosimeter (yearly averaged).

\begin{tabular}{|c|c|c|c|c|c|}
\hline Reseaut & Ctologie & $\begin{array}{l}\text { Activite } \\
\text { cofumique } \\
\text { radon } \\
222 \mathrm{~Bq} \mathrm{m^{3 }}\end{array}$ & 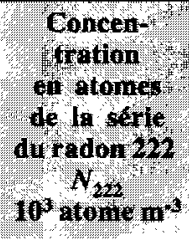 & 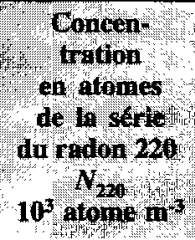 & $\begin{array}{c}\text { Rapport } \\
<\mathbf{A 2 2} / \mathbf{N}_{\mathbf{2 2 0}} \\
\mathbf{N}_{\mathbf{2 n}}\end{array}$ \\
\hline $\begin{array}{c}\text { Bassin } \\
\text { Parisien } \\
\text { France } \\
1990-1995\end{array}$ & $\begin{array}{c}\text { sol } \\
\text { argilo calcaire } \\
\text { ou } \\
\text { argilo sableux }\end{array}$ & 11,2 & 5340 & 16,5 & 324 \\
\hline $\begin{array}{c}\text { Limousin } \\
\text { France } \\
1993\end{array}$ & granites & 20 & 9520 & 6,8 & 1400 \\
\hline $\begin{array}{c}\text { Charentes } \\
\text { France } \\
1992-1993\end{array}$ & $\begin{array}{l}\text { calcaire } \\
\text { sédiments } \\
\text { marins }\end{array}$ & 6,5 & 3100 & 9,2 & 285 \\
\hline $\begin{array}{l}\text { Vendée } \\
\text { France } \\
1993\end{array}$ & $\begin{array}{l}\text { séries } \\
\text { métamor- } \\
\text { phiques } \\
\text { et granitiques }\end{array}$ & 13 & 6200 & 12 & 5170 \\
\hline $\begin{array}{c}\text { Forez } \\
\text { France } 1993\end{array}$ & granites & 11 & 5240 & 10,4 & 504 \\
\hline $\begin{array}{l}\text { Morvan } \\
\text { France } \\
1993\end{array}$ & granites & 17,5 & 8330 & 14,4 & 578 \\
\hline $\begin{array}{l}\text { Languedoc } \\
\text { France } \\
1991\end{array}$ & $\begin{array}{l}\text { sédimentaire } \\
\text { et volcanique } \\
\text { grès permiens }\end{array}$ & 15 & 7140 & 8,3 & 860 \\
\hline $\begin{array}{l}\text { Mounana } \\
\text { Haut Ogoué } \\
\text { Gabon }\end{array}$ & $\begin{array}{l}\text { grès } \\
\text { et } \\
\text { altérations }\end{array}$ & 14 & 6670 & 18,4 & 363 \\
\hline $\begin{array}{c}\text { Arlit } \\
\text { Sud Sahara } \\
\text { Niger }\end{array}$ & $\begin{array}{l}\text { sables } \\
\text { grès }\end{array}$ & 33 & 15700 & 41 & 383 \\
\hline $\begin{array}{c}\text { Kanyemba } \\
\text { Zimbabwe } \\
1991\end{array}$ & $\begin{array}{c}\text { sables } \\
\text { grès }\end{array}$ & 8,5 & 4050 & 13,6 & 298 \\
\hline
\end{tabular}


allant de 300 à 5200 , s'expliquent dans un schéma plus réaliste où les effets du dépôt au sol des aérosols porteurs de descendants du radon sont pris en compte.

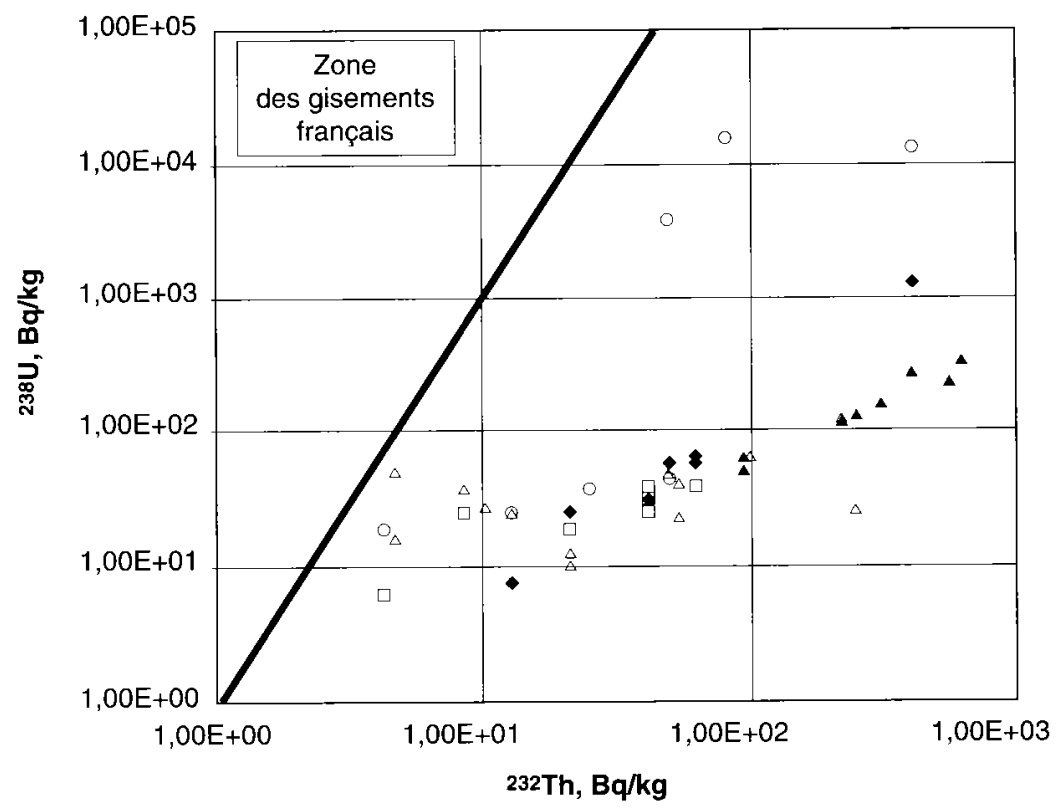

\begin{tabular}{|c|c|c|}
\hline - Ignées [21] & Sédim. [21] & $\square$ Métam. [21] \\
\hline - Ignées | & et [23] & [21] \\
\hline
\end{tabular}

Nota : Les gisements d'uranium français s'interprètent en général par la mobilisation de l'uranium d'une roche mère ignée où l'instauration de conditions oxydantes ont permis à l'uranium de passer de la valence $\mathrm{U}^{\mathrm{VV}}$ peu mobile à la valence $\mathrm{U}^{\mathrm{VI}}$. Le radical uranyl $\mathrm{UO}_{2}$ formé s'insère dans des complexes mobiles tels que le sulfate d'uranyl. Le transport par les eaux souterraines peut l'amener jusqu'à une roche où les conditions géochimiques sont favorables à son piègeage. Le gisement formé est filonien si le piège est un massif fracturé, sédimentaire si le piège est une roche poreuse notamment un grès. Le thorium ne peut pas suivre, car il ne peut pas se mettre sous la valence VI. Partant d'une roche mère où les activités de ${ }^{238} \mathrm{U}$ et ${ }^{232} \mathrm{Th}$ sont du même ordre, on aboutit par ce processus géochimique à un gisement uranifère exempt de thorium.

Fig. 1. - Activités massiques respectives de ${ }^{238} U$ et de ${ }^{232}$ Th dans les roches usuelles.

(Boyle, 1982 ; IAEA, 1990 ; UNSCEAR, 1993)

Respective activities concentrations of ${ }^{238} \mathrm{U}$ and ${ }^{232} \mathrm{Th}$ in common rocks. 


\subsection{Résultats obtenus sur le réseau du Limousin}

La Cogema exploite depuis 45 ans les minerais d'uranium de la région du Nord-Limousin. Les minerais ont été traités sur place, il existe un certain nombre de sources de radon 222, dont des stockages de déchets miniers et de résidus de traitement. L'impact radiologique des émissions de ${ }^{222} \mathrm{Rn}$ est surveillé par un réseau qui comporte 44 stations opérationnelles.

La région couverte par le réseau de surveillance « radon » du Nord Limou$\sin ,\left(50 \mathrm{~km}^{2}\right)$, présente un relief de collines arrondies et boisées, avec des vallées assez marquées et de nombreux lacs. Les stations situées en ligne de crête ont des expositions faibles parce que la dispersion atmosphérique y est forte. Les stations situées en fond de vallée ou protégées par un obstacle ont des expositions fortes parce qu'elles sont mal ventilées.

Elle est caractérisée géologiquement par des formations granitiques, (granites à 2 micas et granites à biotite), avec en bordure des séries métamorphiques, (micaschistes et paragneiss). Au cours de la phase de prospection géologique du Nord-Limousin il a été montré que les teneurs en uranium des sols associés aux granites de la région couverte par le réseau étaient nettement plus élevées que celles observées en moyenne pour des formations plus éloignées. Une campagne aérogammamétrique a permis d'établir une carte des teneurs superficielles des sols en uranium et en thorium. Ces teneurs peuvent être considérées comme étant proportionnelles.

Des campagnes de mesures ponctuelles des flux d'exhalaisons du radon 222 ont permis de produire une carte de ces flux à partir de la carte des teneurs des sols en uranium. Ces campagnes ont montré que les flux locaux sont environ cinq fois plus élevés que les flux obtenus pour des sols associés aux sols des formations sédimentaires plus éloignées.

Ces conditions sont favorables à l'instauration de valeurs du rapport $A$ relativement constantes sur le domaine.

Le tableau II contient les résultats obtenus sur le réseau du Limousin (France) en 1993. Treize stations avaient été identifiées comme stations représentative du bruit de fond. Il donne pour chaque station la valeur moyenne annuelle de la concentration en atomes de radon 222 , en atomes de ${ }^{212} \mathrm{~Pb}$ ou ${ }^{212} \mathrm{Bi}$, du rapport $A$ de ces 2 valeurs, de l'énergie alpha potentielle (EAP) et de l'activité volumique moyenne $A_{\mathrm{v}}$ du radon 222 . On donne aussi le facteur d'équilibre $F$ entre les descendants de ${ }^{222} \mathrm{Rn}$ et le ${ }^{222} \mathrm{Rn}$. Ces moyennes annuelles sont calculées sur les 12 valeurs des expositions mensuelles mesurées en 1993 par les dosimètres actifs alpha spectral mis en service par ALGADE dans les stations de surveillance des sites miniers.

On remarque que les valeurs en moyenne annuelle des activités volumiques de fond du radon 222 mesurées par les 13 stations sont très variables, il y a un 
facteur 2,6 entre la valeur la plus forte valeur, $31,5 \mathrm{~Bq} \mathrm{~m}^{-3}$, station $\mathrm{n}^{\circ} 35$, et la plus faible, $12 \mathrm{~Bq} \mathrm{~m}^{-3}$, station $\mathrm{n}^{\circ} 20$. La technique de restitution des expositions industrielles par soustraction d'une valeur uniforme de l'exposition de fond serait ici inadéquate.

En ce qui concerne les valeurs en moyenne mensuelle des activités obtenues en 1993 par chaque station de « fond », la variabilité est encore plus grande, voir la référence (Bernhard et al., 1995) pour plus d'informations.

\section{Tableau II}

Moyennes annuelles obtenues en 1993 sur les stations de « fond » du réseau Nord-Limousin.

Annual mean values obtained in 1993 at the "background" stations of the Nord-Limousin network

\begin{tabular}{|c|c|c|c|c|c|c|}
\hline $\begin{array}{c}\mathbf{N} \\
\text { des } \\
\text { stations }\end{array}$ & $\begin{array}{l}\text { Rn } 222 \\
\text { atomefim } \\
\times 10^{6}\end{array}$ & $\begin{array}{l}\text { Pb } \\
\text { ou Bi } 2 / 2 \\
\text { atome } / \mathbf{m}^{3} \\
\times 10^{3}\end{array}$ & $\begin{array}{r}4 \\
\times 10^{3}\end{array}$ & $\underset{\mathrm{nJ} / \mathbf{m}^{3}}{222}$ & $\begin{array}{l}A_{\gamma} 222 \\
\mathrm{~Bq} / \mathrm{m}^{3}\end{array}$ & $\boldsymbol{F}$ \\
\hline 8 & 8,5 & 6 & 1,42 & 50 & 17,85 & 0,50 \\
\hline 9 & 8,8 & 6,5 & 1,35 & 54 & 18,48 & 0,52 \\
\hline 16 & 7,1 & 6,8 & 1,04 & 41 & 14,91 & 0,49 \\
\hline 19 & 8,3 & 6,2 & 1,34 & 56 & 17,43 & 0,57 \\
\hline 20 & 5,7 & 4,4 & 1,30 & 43 & 11,97 & 0,64 \\
\hline 23 & 12 & 10 & 1,20 & 57 & 25,2 & 0,40 \\
\hline 27 & 6 & 4,6 & 1,30 & 33 & 12,6 & 0,47 \\
\hline 30 & 6,1 & 4,9 & 1,24 & 38 & 12,81 & 0,53 \\
\hline 31 & 11 & 8,1 & 1,36 & 61 & 23,1 & 0,47 \\
\hline 35 & 15 & 9,9 & 1,52 & 94 & 31,5 & 0,53 \\
\hline 40 & 6,2 & 3,5 & 1,77 & 34 & 13,02 & 0,46 \\
\hline 42 & 13 & 7,8 & 1,67 & 68 & 27,3 & 0,44 \\
\hline \multirow[t]{2}{*}{43} & 15 & 9,4 & 1,60 & 92 & 31,5 & 0,52 \\
\hline & $<222 \mathrm{Rn}>$ & $\left\langle\mathrm{Pb}+{ }^{212} \mathrm{Bi}\right\rangle$ & $\langle A\rangle$ & $<$ EAP $222>$ & $\left\langle A_{v} 222\right\rangle$ & $\langle F\rangle$ \\
\hline $\begin{array}{c}\text { Moyenne } \\
\text { «réseau» } \\
\text { des stations } \\
\text { de fond }\end{array}$ & 9,4 & 6,8 & 1,39 & 55,5 & 19,8 & 0,5 \\
\hline
\end{tabular}

La valeur moyenne de $A$ sur toutes les stations de fond vaut $\langle A\rangle=$ $1,39 \times 10^{3}$, la plus forte valeur étant 1,77 et la plus faible 1,04 . L'écart-type relatif de la distribution des valeurs de $A, \Delta A / A$, est égal à $14 \%$. 
Ces résultats de terrain montrant une faible variabilité des valeurs de $A$, on a considéré que le critère d'applicabilité de la procédure était respecté. Les valeurs de la concentration en atomes de ${ }^{212} \mathrm{~Pb}$ ou ${ }^{212} \mathrm{Bi}$ des stations soumises à l'influence des sources de radon 222 de la COGEMA sont utilisées pour calculer, par multiplication par le facteur $\langle A\rangle$, soit $1,39 \times 10^{3}$, les valeurs des concentrations en ${ }^{222} \mathrm{Rn}$ d'origine naturelle de ces stations.

En soustrayant les valeurs des concentrations en ${ }^{222} \mathrm{Rn}$ d'origine naturelle des valeurs des concentrations cumulées mesurées par les stations soumises à l'influence des sources de radon 222 de la Cogema, on obtient les concentrations en radon 222 d'origine industrielle. Un traitement complémentaire permet de restituer l'EAP 222 de ses descendants. On utilise ici la valeur moyenne du facteur d'équilibre $F$ des stations de «fond», soit 0,5 , voir le tableau II.

$100 \mathrm{~nJ} \mathrm{~m}^{-3}$ en moyenne annuelle correspond à $1 \mathrm{mSv}$ par an.

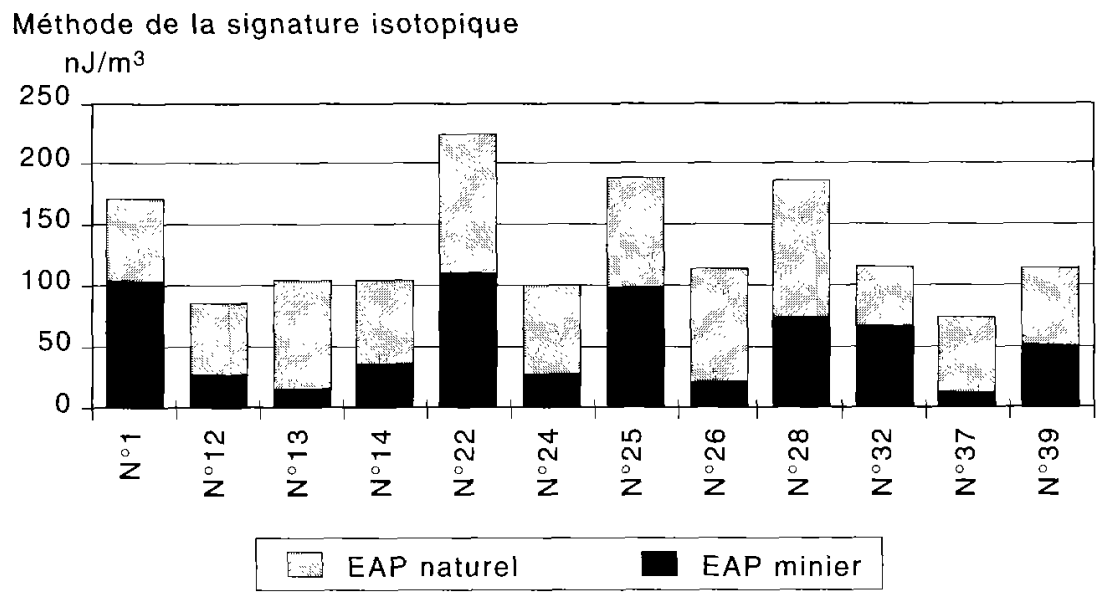

La faible dispersion des valeurs du rapport A sur l'ensemble des stations de fond justifie la méthode de la dilution isotopique dans le cas du réseau du Nord Limousin.

Fig. 2. - Restitution des EAP du radon 222 industriel par la méthode de la signature isotopique. Cas du réseau du Nord Limousin, 12 stations marquées par du radon 222 industriel. Valeurs moyennes de l'année 1993

Recovery of the PAEs of industrial radon 222 by the isotopic signature method. The Nord-Limousin network: 12 stations marked by industrial radon 222. Mean values for 1993.

La figure 2 résume les résultats de cette restitution. Elle fournit, en moyenne annuelle, les valeurs des concentrations en EAP 222 respectivement d'origine industrielle et d'origine naturelle pour les stations de la zone marquée par les émissions de radon 222 des sources Cogema. Certaines de ces sta- 
tions sont implantées au niveau des groupes critiques de la population. On constate que les valeurs d'origine industrielle restent pratiquement toutes inférieures à la limite dérivée de $100 \mathrm{~nJ} \mathrm{~m}^{-3}$, qui correspond sur un an à une dose efficace de $1 \mathrm{mSv}$.

La figure 3 montre les résultats d'une restitution fondée sur la soustraction d'un bruit de fond uniforme sur tout le domaine. On remarque que cette procédure identifie indûment 4 stations où la valeur de la limite dérivée de $100 \mathrm{~nJ} \mathrm{~m}^{-3}$ est dépassée en moyenne annuelle.

$100 \mathrm{~nJ} \mathrm{~m}^{-3}$ en moyenne annuelle correspond à $1 \mathrm{mSv}$ par an.

Méthode de la signature isotopique

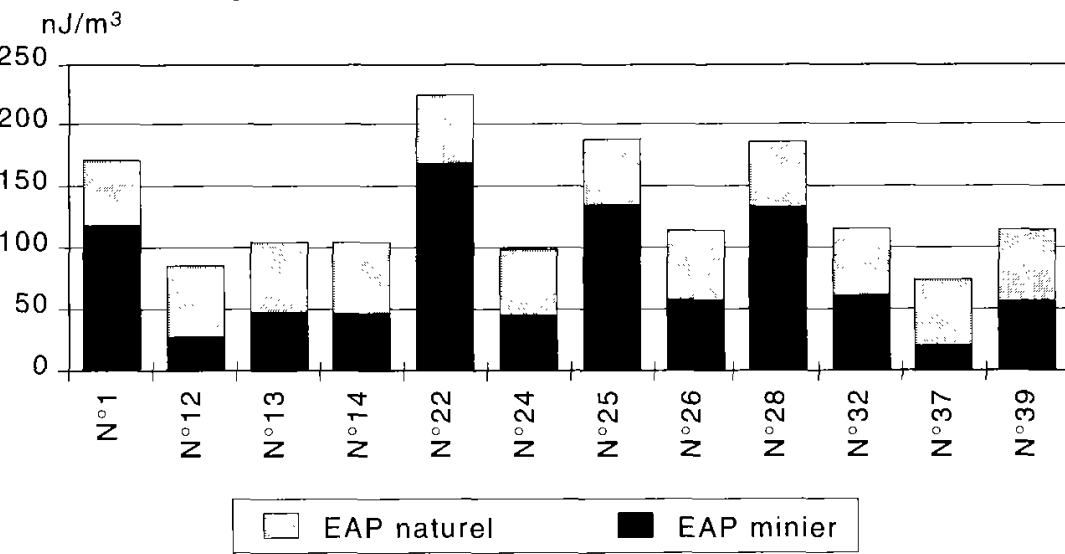

La variabilité des concentrations en EAP 222 mesurées sur les stations de fond disqualifie la méthode de la soustraction d'un bruit de fond uniforme dans le cas du réseau du Nord Limousin.

Fig. 3. - Restitution des EAP du radon 222 industriel par la méthode du bruit de fond uniforme. Cas du réseau du Nord Limousin, 12 stations marquées par du radon 222 industriel. Valeurs moyennes de l'année 1993.

Recovery of the PAEs of industrial radon 222 by the uniform background method. The Nord-Limousin network: 12 stations marked by industrial radon 222. Mean values for 1993.

\subsection{Sélection des stations représentatives $d u$ bruit de fond régional d'origine naturelle}

Nous sélectionnons les stations de fond, sur un réseau supposé assez dense par rapport à la complexité orographique et géologique du domaine, grâce à deux indicateurs, la signature isotopique des descendants à vie courte du radon 222 et l'indice de ventilation. 
On utilise d'abord le fait que le dosimètre alpha spectral fournit la signature des descendants à vie courte du radon 222 . On déduit de cette signature une valeur du facteur d'équilibre $F$ du radon 222 en moyenne mensuelle ou annuelle.

Dans le cas des masses d'air qui passent sur le réseau en n'ayant collecté auparavant que les exhalaisons de sols naturels, la signature isotopique se stabilise. On peut montrer, par une simulation élémentaire, voir (Bernhard et al., 1995), que le facteur d'équilibre $F$, sauf anomalies notables des exhalaisons naturelles locales du ${ }^{222} \mathrm{Rn}$, est certainement supérieur à 0,4 .

Par contre, si la masse d'air vient de se charger à des valeurs significatives des exhalaisons d'un dépôt de résidus, on doit s'attendre à des valeurs de $F$ d'autant plus faibles que le temps de transfert entre ce dépôt et la station est plus court.

En pratique les stations de faibles concentrations en ${ }^{222} \mathrm{Rn}$ pour lesquelles la valeur de $F$ en moyenne annuelle est supérieure à 0,4 sont candidates à être sélectionnées comme station de fond. Celles pour lesquelles la valeur de $F$ en moyenne annuelle est inférieure à 0,2 sont identifiées comme stations certainement marquées par une anomalie locale de ${ }^{222} \mathrm{Rn}$. Il peut s'agir, soit une source industrielle, soit d'un affleurement uranifère encore non exploité.

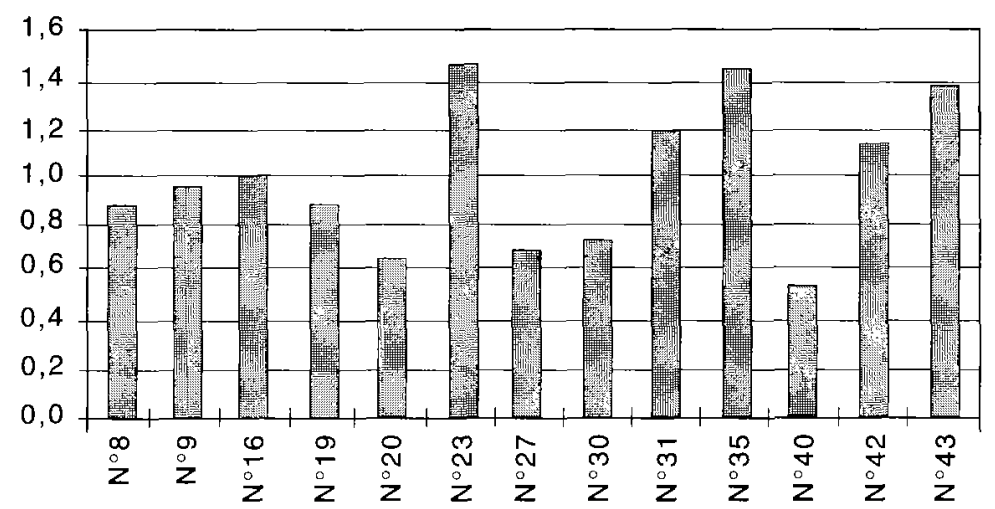

Fig. 4. - Indice de ventilation des 13 stations de fond du réseau Nord Limousin.

Ventilation index of the 13 background stations in the Nord-Limousin network.

Pour chaque station de fond candidate, on définit 1'indice relatif de ventilation comme le rapport de la concentration en atomes de ${ }^{212} \mathrm{~Pb}$ ou de ${ }^{212} \mathrm{Bi}$ qu'on y a mesuré à la valeur moyenne de cette concentration pour toutes les stations du réseau. Le groupe des stations sélectionnées doit bien couvrir l'ensemble des indices de ventilation calculés sur la totalité du domaine sur- 
veillé. Dans le cas du Nord Limousin on a abouti à l'ensemble des indices de ventitation de la figure 4 , qui a semblé représentatif de la variabilité orographique du domaine.

Si l'écart-type relatif des valeurs de $A$ des stations de fond sélectionnées est inférieur à $30 \%$, on peut appliquer la méthode de la signature isotopique.

\subsection{Applications de la méthode de la signature isotopique à d'autres réseaux}

On présente à titre d'exemple sur la figure 5 la restitution effectuée sur le réseau de surveillance du site minier exploité par la Cogema à Lodève (Bernhard et al., 1995), (réseau du Languedoc). En 1991 ce réseau comportait 14 stations environnementales, dont 8 ont été retenues comme stations de fond, avec $\langle A\rangle$ $=860$, l'écart type relatif des valeurs de $A$ étant de $22 \%$.

$100 \mathrm{~nJ} \mathrm{~m}^{-3}$ en moyenne annuelle correspond à $1 \mathrm{mSv}$ par an.

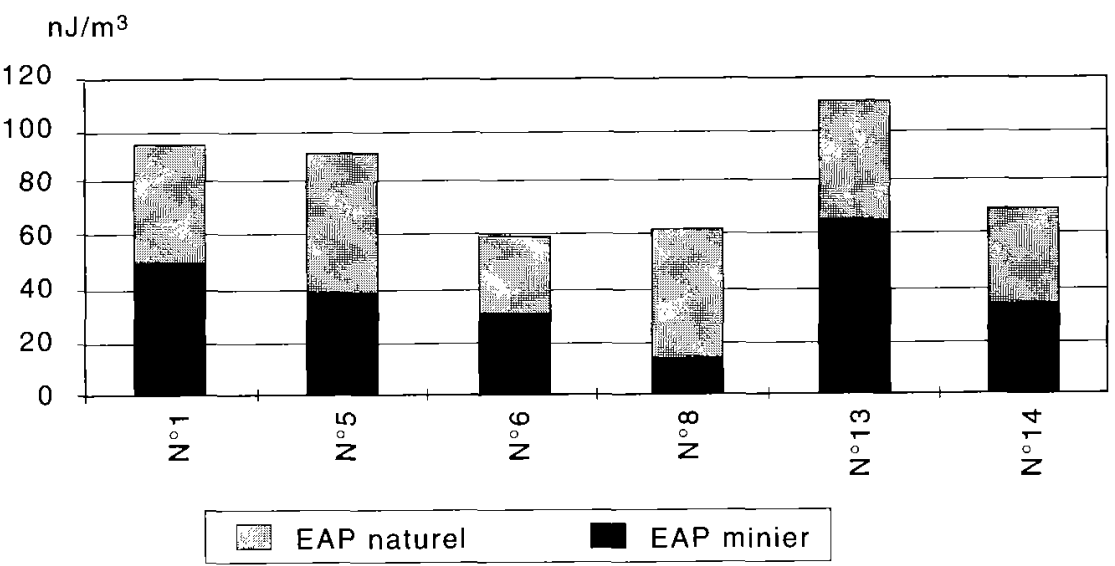

Fig. 5. - Restitution des EAP du radon 222 industriel par la méthode du bruit de fond uniforme. Cas du réseau du Languedoc, 6 stations marquées par du radon 222 industriel. Valeurs moyennes de l'année 1991. Méthode de la signature isotopique.

Recovery of the PAE of industrial radon 222 by the uniform background method. Languedoc network, 6 stations marked by industrial radon 222. Mean values for 1991 . Isotopic signature method. 


\section{La méthode de la signature isotopique dans le cas des ingestions d'uranium et de radium par la voie alimentaire}

Le problème de la discrimination des ingestions d'uranium et de radium d'origine «naturelle » et d'origine « industrielles» par la voie alimentaire se pose dans le cas du massif granitique du Forez (F). La Cogema y a exploité et traité de 1950 à 1980 des minerais d'uranium ayant des teneurs de quelques $\mathrm{kg}$ par tonne, qui ont fourni environ 15000 tonnes de métal. Les résidus de traitement sont stockés en surface sous eau en amont d'une digue de retenue, les plus grenus ayant parfois servi au remblayage des travaux miniers. Un réaménagement substantiel de ce dépôt est envisagé. L'étude préalable de l'impact radiologique de ce réaménagement est prévue. L'ingestion d'uranium et de radium par la voie alimentaire locale est prise en compte pour certains des groupes critiques de ce site. Si la méthode de la signature isotopique est applicable au cas des transferts environnementaux d'uranium et de radium industriels par les eaux souterraines et de surface, elle rendra un grand service sur ce site complexe sur le plan radioécologique.

Dans le cadre de sa mission générale de protection de la population contre les rayonnements ionisants, l'OPRI s'intéresse depuis longtemps à la radioactivité naturelle des eaux minérales. Les résultats des campagnes systématiques d'analyse de la radioactivité des eaux minérales françaises ont été publiées dès 1968 (Rémy et al., 1968). Une nouvelle synthèse des résultats concernant notamment l'abondance relative des isotopes du radium et les déséquilibres des isotopes de l'uranium, et une présentation des méthodes analytiques physiques et radiochimiques de l'OPRI a été publiée en 1990 (Rémy et al., 1990). Les processus physico-chimiques et géochimiques susceptibles d'expliquer l'abondance relative et les déséquilibres radioactifs des isotopes des radionucléides des chaînes de l'uranium et du thorium observés en dissolution dans les eaux minérales ont fait l'objet de la publication (Maisonneuve et al., 1984), ainsi que de la note (Clanet et al.) en ce qui concerne les déséquilibres ${ }^{234} \mathrm{U}$ et ${ }^{238} \mathrm{U}$.

\subsection{Exploitation de la déformation de la signature isotopique de l'uranium du milieu naturel}

Dans les compartiments physiques et biologiques du milieu naturel les isotopes ${ }^{234} \mathrm{U}$ et ${ }^{238} \mathrm{U}$ sont en général assez loin de l'équilibre radioactif. Ils sont par contre en équilibre radioactif dans les effluents industriels. Les performances des méthodes analytiques sont suffisantes pour mesurer le déséquilibre de la signature du milieu naturel, et donc permettent la mise en évidence de la déformation de la signature dans un compartiment de l'écosphère notamment lorsque les composantes « naturelle » et « industrielle » de sa charge radioactive, et donc les doses efficaces associées, sont du même ordre de grandeur. 


\subsubsection{Les déséquilibres ${ }^{234} \mathrm{U}$ et ${ }^{238} \mathrm{U}$ du milieu naturel}

En ce qui concerne les activités des isotopes ${ }^{234} \mathrm{U}$ et ${ }^{238} \mathrm{U}$ de l'uranium dissous dans les eaux souterraines, on sait depuis les publications par Cherdyntsev et al. $(1955 ; 1961)$ qu'elles sont rarement à l'équilibre. Le rapport des activités ${ }^{234} \mathrm{U} /{ }^{238} \mathrm{U}$, noté $A R$, est la signature isotopique. Les résultats publiés dans la littérature (Asikainen, 1981 ; Cothern et al., 1983) montrent que $A R$ est en général supérieur à un. D'après Ferronski et al. (1982), $A R$ vaut de 0,9 à 1,1 dans les eaux des gisements d'uranium, 1,5 à 2,5 dans les eaux des roches sédimentaires et métamorphiques, 2,5 à 4 dans les eaux des roches ignées, et peut atteindre des valeurs de 4 à 5 dans les eaux de Vichy (F), voir Rémy et al., (1990) et Maisonneuve et al. (1984).

Les recherches bibliographiques en cours à l'OPRI (Maggiorella, 1997) ont permis de recenser les modèles phénoménologiques qui rendent compte des évolutions de la signature isotopique de l'uranium observables dans les aquifères souterrains, les sols et les compartiments biologiques.

Plusieurs mécanismes sont proposés qui rendent compte du déséquilibre dans les eaux souterraines. La différence de masse n'explique pas le fractionnement. Un modèle théorique de l'évolution du rapport $A R$ en fonction du temps de contact de l'eau avec la roche est proposé par Andrews et al. (1982). Il avait été utilisé dans le cas des granites de Stripa (Andrews et al., 1978). Ce modèle tient compte de deux mécanismes:

- la différence des taux de lessivage des 2 isotopes, vu les différences de leurs histoires radioactives, minéralogiques et physico-chimiques,

- la capacité d'échappement, hors des matrices cristallines de la roche hôte, des atomes de ${ }^{234} \mathrm{Th}$. Ces atomes, qui descendent de ${ }^{238} \mathrm{U}$ par décroissance alpha, ont une énergie de recul suffisante pour avoir des parcours de quelques dizaines de nanomètres dans les réseaux cristallins (Hongbin Sun, 1995). Ils peuvent donc passer en phase aqueuse s'ils sont assez proches de la surface du grain uranifère.

Le temps de référence pour le temps de contact est la période radioactive de ${ }^{234} U, 2,45 \times 10^{5}$ ans. Pour des temps courts, $A R$ est égal au rapport des taux de lessivage. Ce n'est que pour des temps longs et dans le cas des minéralisations uranifères de type filonien que le mécanisme de l'échappement des atomes de ${ }^{234}$ Th par énergie de recul peut prendre le dessus. Les perspectives de la datation des eaux souterraines à l'aide des isotopes de l'uranium sont examinées par Frölich et al. (1987).

La note (Clanet et al.) avait pour objet d'expliquer le déséquilibre des isotopes ${ }^{234} \mathrm{U}$. et ${ }^{238} \mathrm{U}$ par le fait que $U$ peut passer de la valence 4 à la valence 6 par des processus radiochimiques lors des désintégrations radioactives qui font passer en 3 étapes ( 1 émission alpha et 2 émissions bêta) l'ion uranium de la masse 238 à la masse 234. L'uranium est plus soluble sous la valence 6 que sous la valence 4 . Cette note s'appuyait sur les résultat des mesures faites 
sur une nappe phréatique située en terrain granitique dans la région du Forez, (F). Le rapport $A R$ des activités dissoutes valait 2,09, résultat annoncé avec une erreur relative de $5 \%$, le granit aquifère étant lui-même à l'équilibre.

Dans les sols les processus sont complexes, faisant intervenir la nature des sols, les vitesses d'érosion et les conditions oxydantes ou réductrices du lessivage des différents horizons. Suivant les cas $A R$ est très inférieur ou très supérieur à 1 dans les particules des sols, la situation étant inversée dans les eaux de ruissellement.

\subsubsection{Signature isotopique de l'uranium relâché en phase aqueuse par les installations minières}

Les déchets d'extraction des exploitations de minerais d'uranium, contenant de l'uranium à des teneurs sub-économiques de quelques grammes par tonne à 200 grammes par tonne, sont stockés en surface. Ils peuvent aussi contenir des pyrites, ce qui conduit en milieu oxygéné à la possibilité du drainage acide des haldes, et à la solubilisation in situ des traces d'uranium. Ce processus peut également concerner les résidus de traitement stockés à l'état humide, et à la solubilisation in situ de la fraction de l'uranium qui n'a pas été extrait en usine. Il y a aussi bien souvent en surface des installations de lixiviation en tas des minerais pauvres, qui à long terme peuvent fuir. La signature isotopique de l'uranium de tous ces rejets en phase liquide est $A R=1$.

\subsection{Exploitation de la déformation de la signature isotopique du radium du milieu naturel}

Les processus minéralogiques, radiochimiques, géochimiques et d'adsorption qui expliquent l'abondance relative des isotopes du radium dans les eaux souterraines et dans les eaux de surface ont bien été identifiés par les prospecteurs d'uranium et les spécialistes de la datation par les méthodes isotopiques. L'influence de la teneur des eaux en anions (chlorures, carbonates, sulfates), et du potentiel d'oxydoréduction est mise en évidence dans (Maisonneuve et al., 1984). La signature isotopique fait intervenir non seulement ${ }^{228} \mathrm{Ra}$ et ${ }^{226} \mathrm{Ra}$, mais aussi ${ }^{224} \mathrm{Ra}$ et ${ }^{223} \mathrm{Ra}$. L'essentiel de la signature est toutefois le rapport des activités du ${ }^{228} \mathrm{Ra}$ et du ${ }^{226} \mathrm{Ra}$. Dans les milieux naturels ce rapport ne s'écarte pas notablement du rapport des activités du ${ }^{232} \mathrm{Th}$ et de ${ }^{238} \mathrm{U}$ des roches mères, indiquant des processus de lessivage de même efficacité quelque soit l'isotope du radium. Dans les effluents aqueux des installations minières françaises, l'activité du ${ }^{228} \mathrm{Ra}$ est très faible en comparaison de celle du ${ }^{226} \mathrm{Ra}$.

Les performances des méthodes analytiques sont suffisantes pour mesurer le rapport des activités du ${ }^{228} \mathrm{Ra}$ et du ${ }^{226} \mathrm{Ra}$ des milieux naturels, et d'établir l'occurrence des enrichissements en ${ }^{226} \mathrm{Ra}$ des milieux récepteurs des effluents industriels. 
UTILISATION DE LA SIGNATURE ISOTOPIQUE DES RADIONUCLÉIDES...

La méthode de la signature isotopique du radium a été employée avec succès par les auteurs du rapport (Martin et al., 1996) pour étudier le comportement du ${ }^{226} \mathrm{Ra}$ relâché dans l'aquifère souterrain par le soubassement d'un dépôt de résidus de traitement d'uranium en Australie.

\section{Conclusions}

\subsection{Restitution des doses efficaces dues à l'EAP 222 inhalée}

Sur les sites français les doses efficaces annuelles dues à l'EAP 222 inhalée d'origine «naturelle » sont dans la gamme allant de 0,5 à $1 \mathrm{mSv}$, celles d'origine «industrielle» sont légalement inférieures à $1 \mathrm{mSv}$.

Nous retenons les points suivants qui justifient la mise en auvre de la méthode des signatures isotopiques pour établir les doses dues à l'EAP 222 « industrielle» de certains sites d'orographie complexe :

- L'expertise des résultats du réseau du Nord Limousin a montré que si la restitution est basée sur la soustraction d'un bruit de fond uniforme sur tout le domaine, les concentrations moyennes en EAP sont sous-estimées pour les stations mal ventilées et surestimées pour les stations bien ventilées. Il en résulte, pour les sites où le bruit de fond est variable, des possibilités de litige entre l'exploitant, l'administration et les association de défense de l'environnement, ceci d'autant plus qu'un certain nombre de valeurs de fond dépasserait la limite

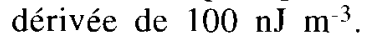

- La méthode de la signature isotopique peut fournir la solution au problème de la restitution correcte des valeurs industrielles si le rapport $A$ reste constant sur les stations de fond du réseau. Il faut toutefois remarquer que l'opération de sélection du sous ensemble des stations considérées comme les stations de fond du réseau est une opération cruciale et délicate. Ce sous ensemble, d'une part doit avoir été sélectionné pour représenter toutes les conditions de ventilation du domaine, d'autre part ne doit comprendre que des stations certainement non marquées par les sources industrielles ou par des anomalies locales des exhalaisons de radon 220 ou de radon 222.

- La méthode ne peut fonctionner que si le nombre de stations est suffisant et si leurs implantations répondent aux exigences énoncées plus haut. Un certain nombre des réseaux français en exploitation sont à densifier dans cette perspective.

Il est envisagé que les réseaux de surveillance des sites miniers français, à forte densité de stations, soient intégrés dans des réseaux moins denses mais représentatifs à l'échelle régionale, qui seront équipés des mêmes matériels de dosimétrie alpha spectrale active, et gérés par l'OPRI. 


\subsection{Restitution des doses efficaces dues à l'ingestion d'uranium}

Sur les sites français les doses efficaces annuelles dues à l'ingestion d'uranium d'origine «naturelle» sont dans la gamme 0,05 à $0,1 \mathrm{mSv}$. Celles d'origine « industrielle » sont légalement inférieures à $1 \mathrm{mSv}$, et en pratique du même ordre de grandeur que celles d'origine naturelle.

Nous retenons les 2 points suivants, favorables à la mise en cuvre de la méthode de la signature isotopique dans le cas des marquages des eaux souterraines par l'uranium industriel :

- Les eaux des aquifères souterrains présentent des rapport des activités $A R={ }^{234} \mathrm{U} /{ }^{238} \mathrm{U}$ en général supérieur à un, mesurables avec une bonne précision dans un laboratoire spécialisé,

- Les isotopes de l'uranium sont à l'équilibre dans les minerais et dans les effluents liquides des usines.

La difficulté de l'entreprise peut venir de ce que les eaux qui ont percolé dans les installations de surface de la mine ou de l'usine sont aussi chargées en sels minéraux, tels que des chlorures et des sulfates, avec des $\mathrm{pH}$ acides ou basiques. Des processus géochimiques complexes peuvent rendre ardue l'interprétation quantitative des modifications de la signature isotopique des eaux naturelles. La charge chimique des eaux de drainage des dépôts de surface peut en effet à elle seule conduire à l'augmentation du lessivage naturel de l'uranium des roches aquifères du soubassement.

\subsection{Restitution des doses efficaces dues à l'ingestion de ${ }^{226}$ Ra}

Sur les sites français les doses efficaces annuelles dues à l'ingestion de ${ }^{226} \mathrm{Ra}$ d'origine « naturelle » sont dans la gamme 0,05 à $0,1 \mathrm{mSv}$. Celles d'origine « industrielle» sont légalement inférieures à $1 \mathrm{mSv}$, et en pratique du même ordre de grandeur que celles d'origine naturelle.

Nous retenons les 2 points suivants, favorables à la mise en œuvre de la méthode de la signature isotopique dans le cas des marquages des eaux souterraines par le radium industriel :

- À l'échelle d'un bassin versant les eaux souterraines et les eaux de surface, chacune en ce qui la concerne, ont une signature isotopique radium ( ${ }^{228} \mathrm{Ra}$, ${ }^{226} \mathrm{Ra}$ et ${ }^{224} \mathrm{Ra}$ ) identifiable avec précision avec les moyens analytiques dont dispose l'OPRI.

- Les effluents liquides de mines et d'usine, et les eaux de percolation des dépôts de surface radifères ne rejettent dans l'environnement que ${ }^{226} \mathrm{Ra}$. 


\section{RÉFÉRENCES}

Andrews J.N. and Kay R.L.F. (1978) The Evolution of enhanced ${ }^{234} \mathbf{U} /{ }^{238} \mathrm{U}$ ratio for dissolved uranium and groundwater dating, Fourth Int. Conf. Geochronology and Isotope Geology, Denver USA, US Geol. Surv. Open File Report, 78-701, p. 11.

Andrews J.N., Giles I.S., Kay R.L.F. and Lee D.J. (1982) Radioelements, radiogenic helium and age relationship for groundwaters from the granites at Stripa, Sweden, Geochimica et Cosmochimica Acta, 46, 1513-1543, Pergamon Press Ltd, USA.

Asikainen M. (1981) State of disequilibrium between 238U, 234U, 226Ra, and 222Rn in groundwater from bedrock, Pergamon Press Ltd, Geochimica at Cosmochimica Acta, 45, 201-206.

Bernhard S., Y. Vauzelle and Zettwoog (1992) Dosimetric Monitoring of the Radon Risk to Populations near Mines, lnt. Conf. on Radiation Protection in Uranium Mines, Int. Conf. on Radiation Protection in Uranium Mines, Saskatoon, Saskatchewan, Canada, 25-28. Edited by Uranium Saskatchewan, Saskatoon, Canada.

Bernhard S., Y. Vauzelle and Zettwoog (1995) Measurement of the radiological impact of environmenal radon 222 releases from heaps of solid wastes from the mineral industry, IRPA endorsed Int. Conf. on Radioprotection and Radioactive Waste Management in the Mining and Mineral Industries, Johannesburg, South Africa, 20-24.

Boyle R.W. (1982) Geochemical prospecting for thorium and uranium deposit, Development in economic geology, 16, Ed. Elsevier Scientific Publishing Company, 1-25.

Cherdyntsev V.V., Chalov P.J. et Khaidarov G.Z. (1955), Izv. Akad. Nauk, SSSR, p. 175.

Cherdyntsev V.V., Orlov D.P., Isabaev E.A. et Ivanov V.I. (1961) Geokhimia, p. 840.

Clanet F., Leclerc J., Rémy M.L. et Moroni J.P. Mise en évidence expérimentale du rôle de l'absorption différentielle du thorium et de l'uranium sur les roches silicatées dans l'état d'équilibre entre les activités ${ }^{234} \mathrm{U}$ et ${ }^{238} \mathrm{U}$ dans la nature, C.R.A.S., 282, série D, 807-810.

Cothern C.R. and W.L. Lappenbusch (1983) Occurence of uranium in drinking water in the U.S., Health Physics, 45, 89-99.

Ferronsky V.I. and Polyakof V.A., translated by S.V. Ferronsky (1982) Environmental isotopes in the hydrosphere, Ed. A. Wiley Interscience Publication, pp. 324-350.

Frölich K. and Gellerman R. (1987) On the potential use of uranium isotopes for grounwater dating, Chemical Geology (Isotope Geoscience Section), 65, 65-77, Elsevier Science Publishers B.V.

Hongbin Sun (1995) Monte Carlo Simulation of Radon Emanation from dry building Materials, Health Physics, 68, n०4.

IAEA (1990) The Environmental Behaviour of Radium, TRS, No 310, 1, p. 44, table XV, Vienna, Austria.

IAEA (1975) Objectives and Design of Environmental Monitoring Programmes for Radioactive Contaminants, Safety Series, 43, Vienna.

ICRP (1977) Recommendations of the ICRP, ICRP Publication 26, Pergamon preess, Oxford, New York, Frankfürt.

ICRP (1984) Principles of Monitoring for the Radiation Protection of the Population, ICRP Publication 43, Pergamon press, Oxford, New York, Frankfürt.

Maggiorella (1997) Rapport interne OPRI.

Maisonneuve J. et Rémy M.L. (1984) Les éléments radioactifs naturels et les eaux thermo-minérales, Congrès sur les données actuelles sur la radioactivité naturelle, SFRP, Monte-Carlo, 5-7.

Martin P. et Akber R.A. (1996) Groundwater seepage from the uranium mine tailings dam : Radioisotopes of radium, thorium and actinium, published by Supervising Scientist, 40 Blackall Street, BARTON, ACT 2600, Australia.

Rémy M.L. et Pellerin P. (1968) Radioactivité naturelle de 250 sources hydrominérales françaises, rapport SCPRI nं 117, Bull. INSERM, 23, (1), 23-62.

Rémy M.L. et Lemaitre N. (1990) Eaux minérales et radioactivité, Hydrogéologie $n^{\circ} 4,267-278$.

UNSCEAR (1993) Sources and effects of ionising radiation, New York. 